\title{
Relative humidity FOS design using spherical hydrogel on no-core multimode fiber
}

\author{
Aida Nasiri ${ }^{1}$, Somayeh Makouei $^{{ }^{*}}$, Tohid Yousefi Rezaii ${ }^{1}$ \\ ${ }^{11^{*}}$ Faculty of Electrical and Computer Engineering, University of Tabriz, Tabriz, 51664, Iran \\ *corresponding author, E-mail: makouei@tabrizu.ac.ir
}

\begin{abstract}
In this paper, design of a new combined optical fiber-based relative humidity $(\mathrm{RH})$ sensor is introduced, which is formed by spherical hydrogel droplets over a no-core fiber. The designed sensor is able to detect humidity changes based on Evanescent waves and splice losses. Refractive index of hydrogel spheres consisting of PEGDMA droplets changes with variation of humidity and leads to intensity changes in the fiber. The sensor structure includes a no-core fiber in length of $4 \mathrm{~mm}$ coupled with two multimode fibers which performs sensing by three hydrogel spheres with diameter of $1 \mathrm{~mm}$. Simulation results show that the more the coupling length and the spherical diameter are, the less influence is achieved for the sensor sensitivity. Therefore, useful advantages are provided for the designed structure in addition to some associated with the two primary sensors, including the reduction of the length of sensing portion and also an increase in the sensitivity and the range of humidity detection. The obtained sensitivity is about $0.13643 \mathrm{~mW} /$ $\% \mathrm{RH}$ in humidity range of 20 to $95 \% \mathrm{RH}$.
\end{abstract}

\section{Introduction}

Today, in the industrial world, humidity is an effective parameter in protecting the environment, meteorology, warehousing, industry, agricultural and medical products, so, it is a very important physical quantity. Humidity is a term used to describe the amount of water vapor found in a gas mixture, and relative humidity (RH) can also be defined as the ratio of the actual water vapor partial pressure in the air mixture to the saturated water vapor pressure at the given temperature [1]. Despite the application of different types of humidity sensors in the industry, the comprehensive comparison of sensors and the study of their problems and shortcomings have been considered. A wide range of types of humidity sensors are divided into three categories: electronic, acoustic, and optical [2]. With the advent of the optical fiber technology, a significant share of researches on humidity sensing has been devoted to optical fiber-based methods. The optical fiber-based humidity sensors are secondary devices against electronic and mechanical counterparts, but show applicable features such as remote or multi-sensing capability [3,4]. Their small size and inexpensive fabrication make an accessible and affordable measurement in limited areas with small dimensions. There are also important features such as high resistance and safety against electromagnetic interference which the electronic humidity sensors cannot support them [5]. These important and practical benefits of optical fiber, recently, make the number of scientists who carry out extensive research on FObased humidity sensors steadily increasing [6-9]. Furthermore, the intensity-based optical fiber sensors offer a powerful combination of simplicity and low cost compared to examples of optical fibers based on fiber Bragg gratings [10], long period gratings [11], photonic crystal fiber interferometers $[12,13]$ and so on.

Various techniques for measuring relative humidity $(\mathrm{RH})$ have been reported using intensity-based fiber optic sensors, all of which are based on evanescent waves. Basically, function of these sensors is based on the interaction of evanescent waves (EW) with the surrounding medium. Changes in the transmitted light due to the interacting EW waves make the output power variations which is detected as intensity changes in fiber. Different methods have been used for the intensity-based humidity sensors that work based on changes of refractive index of humidity responsive materials. Furthermore, among the sensitive materials which perform an important role in humidity sensing, hydrogels are more beneficial. These gels which contain water but cannot dissolve in it, present useful mechanical and biomedical superiorities. For instance, hydrogels have a wider dynamic range for humidity sensing in a shorter response time due to their bigger irregular pores which can be controlled for reducing the cross-sensitivity. They also, particularly, contain a high light transparency and mechanical strength [14]. Accordingly, by changing the refractive index of the applied hydrogel due to humidity variations, the intensity of optical fiber will be changed. The difference between existing methods is due to the creation of different structures to increase the efficiency of the evanescent waves, including the tapered optical fibers [15], hetero-core optical fiber [16] and U-shaped bending optical fibers [17,18]. Among the various methods, two of which introduce new structures of intensity-based fiber optic sensors are as the pattern of designed humidity sensor in this article. In [19] a new form of cladding is proposed and designed in the sensor area. One or more drops of poly (ethylene glycol) dimethacrylate (PEGDMA) are used as hydrogel sphere coatings instead of an even-thickness coating on the fiber bare core which has shown the important properties [20,21]. The refractive index 
of the hydrogel is greater than the fiber core, and as the refractive index increases, the humidity decreases. Also, the transmission ratio of the fiber is reduced at once by increasing the refractive index in a certain range of humidity and the sensing operation begins (in a refractive index greater than 1.43). The reduction of this power transmission is influenced by the changes in the number, size, and distance between the spheres. The results have been investigated for each one, and the number of spheres has had a significant effect on the relative humidity and sensor sensitivity. In [22], the no-core structure is presented among two single-mode optical fiber. In this humidity sensor, a hydrogel coating structure of HEC / PVDF is deposited on no-core fiber. The sensor sensitivity for $2 \mathrm{~cm}$ length of no-core is $0.196 \mathrm{~dB} / \%$ $\mathrm{RH}$ and the linear response is obtained in the humidity range of 40 to $75 \% \mathrm{RH}$.

In this article, the designed sensor has a no-core fiber structure that forms a splice loss which is attached with hydrogel spheres to create more sensitivity. First of all, the detailed structure of stimulated sensor that is captured from two above mentioned sensors will be discussed. Next, it is intended to accomplish an appropriate behavior by adjusting the structural parameters and investigating all aspects of the combined sensor that not only has received the advantages of the two previous methods, but also has shown better specifications. At the end of the article, the ultimate optimized structure is proposed by comparing the achieved results from all stimulations. Finally, the paper is ended by a brief conclusion.

\section{Sensor structure}

The structural properties of the proposed sensor are presented in this section. Fig. 1 shows the basic structure of this sensor which includes a no-core fiber coupled with two multimode fibers and the same hydrogel spheres as those depicted in the figure for more sensitivity. The multimode fibers have the Silica core and cladding in diameter of $60 \mu \mathrm{m}$ and $125 \mu \mathrm{m}$ and their refractive indexes are 1.4517 and 1.40 respectively. The diameter of the no-core fiber is $125 \mu \mathrm{m}$, which forms the sensing part. The multimode fibers act as optical waveguides in the sensor structure. Refractive index of the spherical hydrogel varies from 1.40 to 1.51 by alternating the relative humidity of the chamber from 100 to $20 \% \mathrm{RH}$. The temperature of the chamber is maintained at $30^{\circ} \mathrm{C}$ during the humidity variations and the fabrication process is similar to the experimental report in the article [19] for the no-core fiber. Also, LED connection is used as a light source with wavelength of $631 \mathrm{~nm}$. In order to simple investigation of the sensor operation, some physical parameters are defined. The $L, d, a, x$, and $N$ are the length of no-core fiber, diameter of spherical hydrogel, distance between center of first spherical hydrogel from the beginning of no-core fiber, separation distance between center of spherical hydrogel, and the number of spheres respectively.

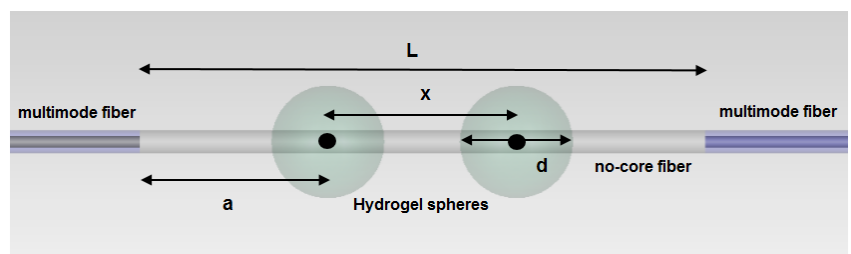

Figure 1: The proposed structure of sensor.

By eliminating the core, part of the power associated with the evanescent wave is increased. Therefore, when light propagates through the no-core fiber, a kind of splice losses occur at the point where two fibers are bonded, so, some modes of the transmitted light in multimode fiber are transferred to the no-core fiber modes which arises from the failure of adaptation to the mode field. Same as the first point of bonding, at the second one, some of the propagated modes are converted into core modes of multimode fiber. Here is a humidity-sensitive hydrogel in solid form of (polyethylene glycol) dimethacrylate (PEGDMA) which has been used by [19]. At first, the spherical form of applied hydrogel, suddenly, makes the ratio of output power to be deduced but after increasing in the refractive index of hydrogel sphere, the transmission of light is enhanced gradually, which is shown in Fig. 2. Then hydrogel sphere encloses more leaking modes in higher refractive index which is more than the refractive index of no-core. Thus, by more absorption of evanescent tail that is traveling out from the no-core fiber, it can improve the coupling power of light to another multimode fiber.

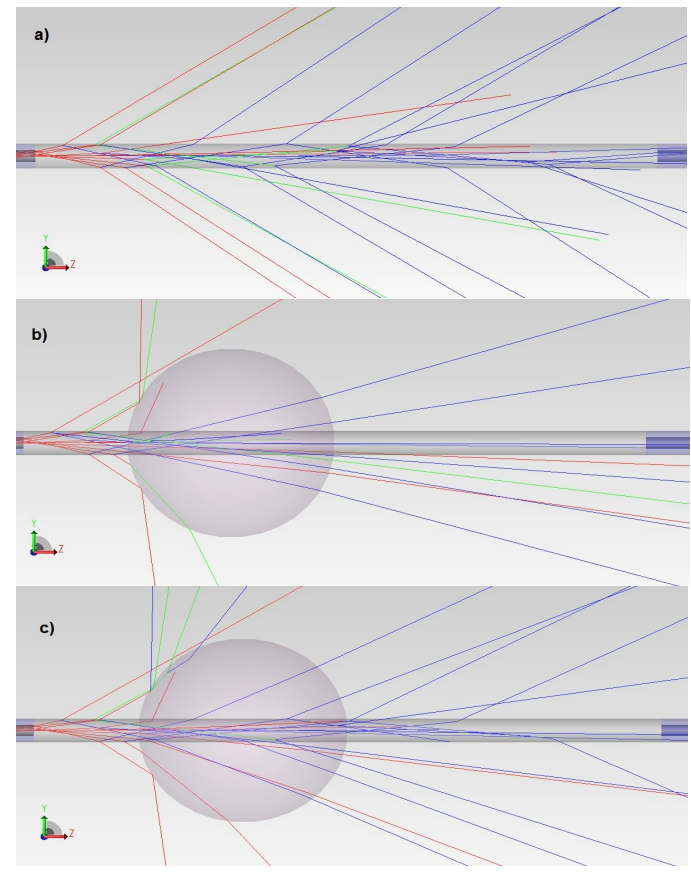

Figure 2: Illustration of the transmitted light for three situations of sensor to show the influence of hydrogel sphere on sensor function. a) Without hydrogel sphere, b) with a hydrogel sphere with refractive index of 1.4 , c) with a hydrogel sphere with refractive index of 1.51. Color of light rays represent the intensity range. 


\section{Simulation results and Discussion}

In this section the effect of physical parameters on the sensor operation which is defined in Fig. 1, is investigated. In all simulations for investigating the sensitivity of the sensor, the ratio of variations in output power related to refractive index changes of hydrogel sphere is presented. Finally, the obtained results from the outputs will be compared. The aim of these parametric studies is to achieve the best optimized sensitivity for the wide range of relative humidity as the sensor input. In the meantime, the linear response of the sensor must be taken into account. To inspect the sensor output power due to hydrogel refractive index variations, firstly, a central point for simulation is considered. The initial values for the sensor structural parameters are as follows; $L$ $=2 \mathrm{~mm}, a=0.3 \mathrm{~mm}, d=0.6 \mathrm{~mm}$, and $N=1$. It is clear that $x$ parameter is used in the cases with more than one spherical hydrogel.

\subsection{The effect of the location of sphere (a parameter)}

First of all, the effect of $a$ parameter is examined. Fig. 3 shows the output power of the fiber by increasing the refractive index of the hydrogel from 1.4 to 1.51 when the sphere is displaced from the beginning of the no-core to its end with the step amount of $0.2 \mathrm{~mm}$. The highest variation of output power occurs at $a=0.9 \mathrm{~mm}$, and the slope of curves are reduced in other circumstances.

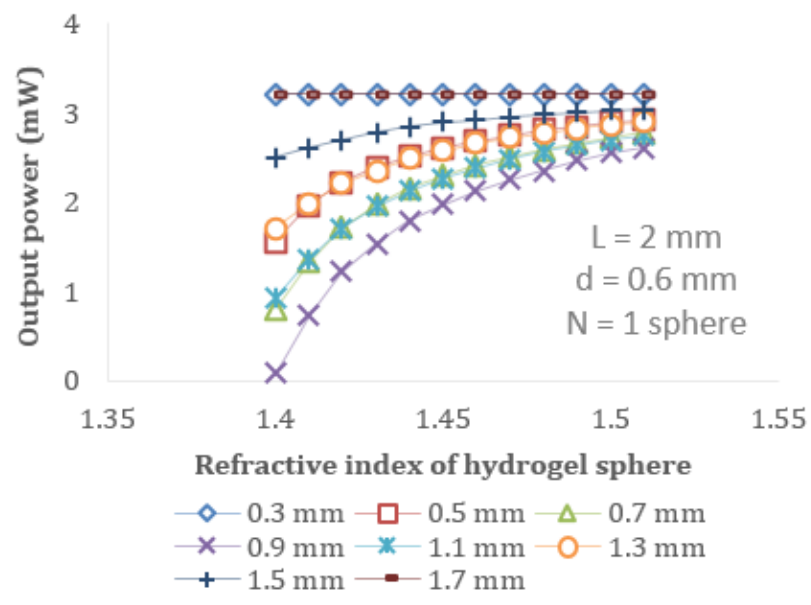

Figure 3: Sensor output power $(\mathrm{mW})$ vs. refractive index of hydrogel as a function of $a$ parameter.

In Fig. 4, the effect of the location of sphere is examined with a change in the no-core fiber length to $5 \mathrm{~mm}$. Thus, by comparing the curves obtained from the displacement of the sphere with the step value of $0.6 \mathrm{~mm}$, it can be seen that the maximum variation of output power remains at $a=0.9 \mathrm{~mm}$. Also, by comparing the results presented in Fig. 3 and 4, it is remarkable that the variations of output power with the displacement of the sphere over the no-core fiber in the length of $2 \mathrm{~mm}$ is more than the length of $5 \mathrm{~mm}$.

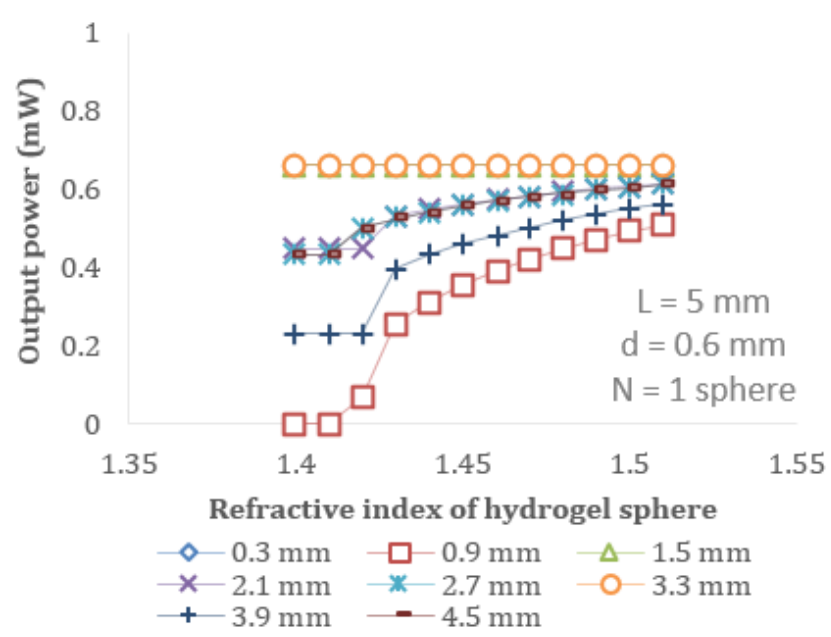

Figure 4: Sensor output power (mW) vs. refractive index of hydrogel as a function of $a$ parameter for $5 \mathrm{~mm}$ length of nocore fiber.

\subsection{The effect of the no-core length ( $L$ parameter)}

Another case to be investigated is the length of the no-core fiber. If the interactive length of the no-core is defined as $L$, the changes in output power with boosting from the length of $1 \mathrm{~mm}$ have been studied. Fig. 4 shows the output power of the fiber by increasing the refractive index of the hydrogel when the length of no-core fiber changes. It can be clearly seen from Fig. 5 that there is no regular changes in the output power for different lengths, but there is only a high sensitivity in length of $2 \mathrm{~mm}$ and a definite deduction in sensitivity through increasing the length of no-core fiber after $5 \mathrm{~mm}$. It is noteworthy that because of limitation in length of $1 \mathrm{~mm}$, the structural parameter $a$, is changed in this length and decreased from $0.9 \mathrm{~mm}$ to $0.5 \mathrm{~mm}$ presenting a very low sensitivity.

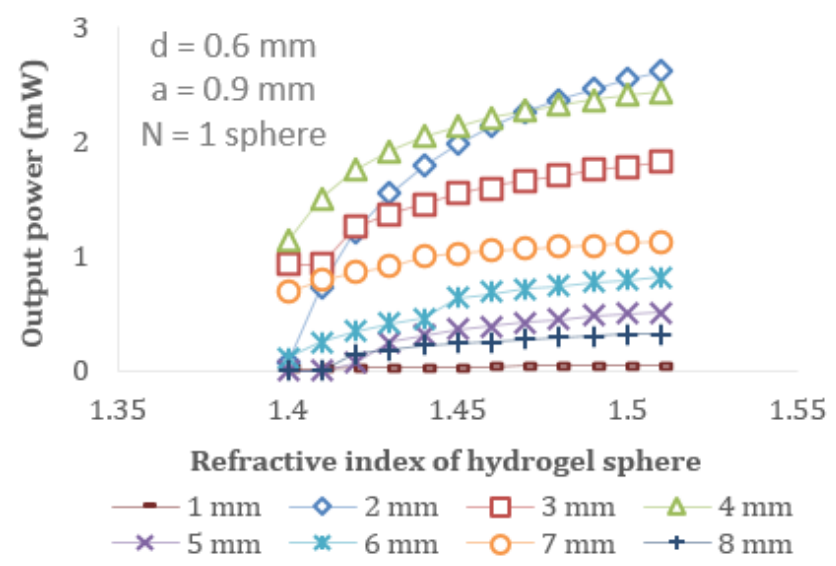

Figure 5: Sensor output power $(\mathrm{mW})$ vs. refractive index of hydrogel as a function of $L$ parameter.

\subsection{The effect of the sphere diameter ( $d$ parameter)}

Although it is expected that as the diameter of sphere increases, more leakage modes will be affected in order to improve the sensitivity of the sensor, but according to Fig. 6, it is clear that increasing the diameter of hydrogel spheres is 
not effective on the sensitivity. Although the larger spheres cover more parts of the length of no-core, they ultimately produce the same sensitivity as sphere with diameter of 0.6 $\mathrm{mm}$. Therefore, it can be said that other parts of the fiber, other than the one that has the most light leakage $(a=0.9 \mathrm{~mm})$, will not affect the sensitivity of the sensor due to the evanescent field span and its depth in this area.

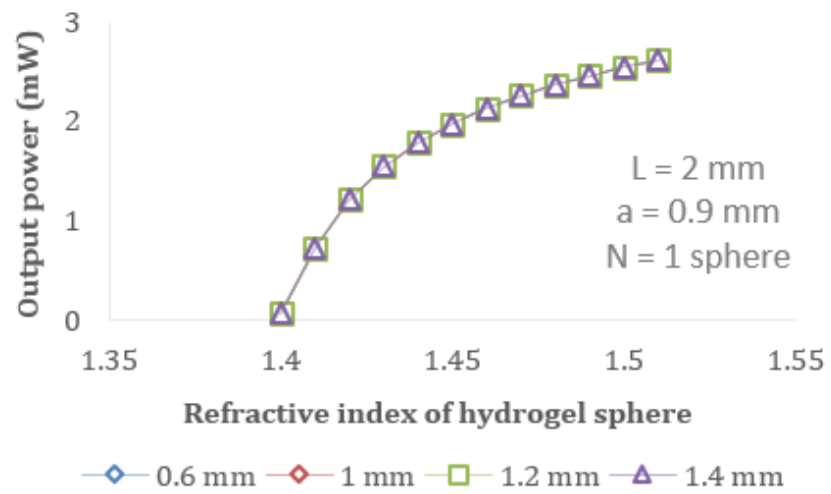

Figure 6: Sensor output power ( $\mathrm{mW})$ vs. refractive index of hydrogel as a function of $d$ parameter for $2 \mathrm{~mm}$ length of nocore fiber.

For more investigation of $d$ parameter influence, the same study is carried out for the no-core fiber in length of $4 \mathrm{~mm}$. As it can be seen from the diagrams of Fig. 7, the only observed difference in the results of two different lengths is that the sensitivity in length of $2 \mathrm{~mm}$ is the same for all sizes of sphere. But as the diameter of sphere increases from 0.6 to $1 \mathrm{~mm}$, the sensitivity grows slightly for length of $4 \mathrm{~mm}$ (it is more perceptibly in high humidities) and then stays constant.

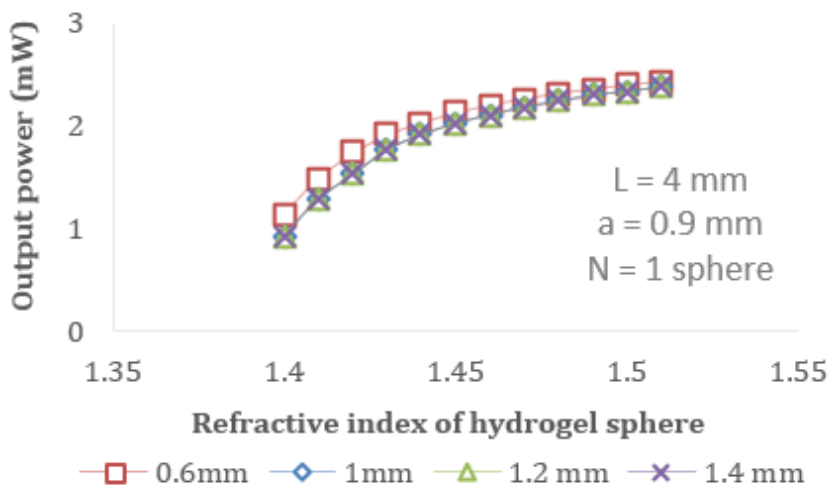

Figure 7: Sensor output power $(\mathrm{mW})$ vs. refractive index of hydrogel as a function of $d$ parameter for $4 \mathrm{~mm}$ length of nocore fiber.

According to the results of Fig. 3, a sphere with the diameter of $0.6 \mathrm{~mm}$ shows only the highest sensitivity at $a$ $=0.9 \mathrm{~mm}$, and with a slight displacement of $0.1 \mathrm{~mm}$, its sensitivity is decreased. Therefore, the acceptable range for the displacement of the sphere with no alternation in the sensitivity for the sensor is also examined for 0.8 and $1 \mathrm{~mm}$ in Fig. 8.

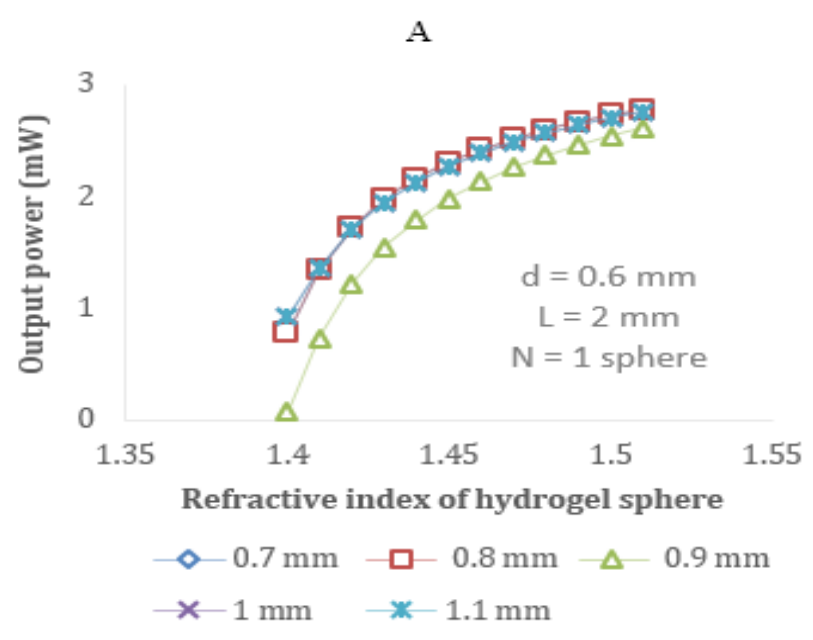

B

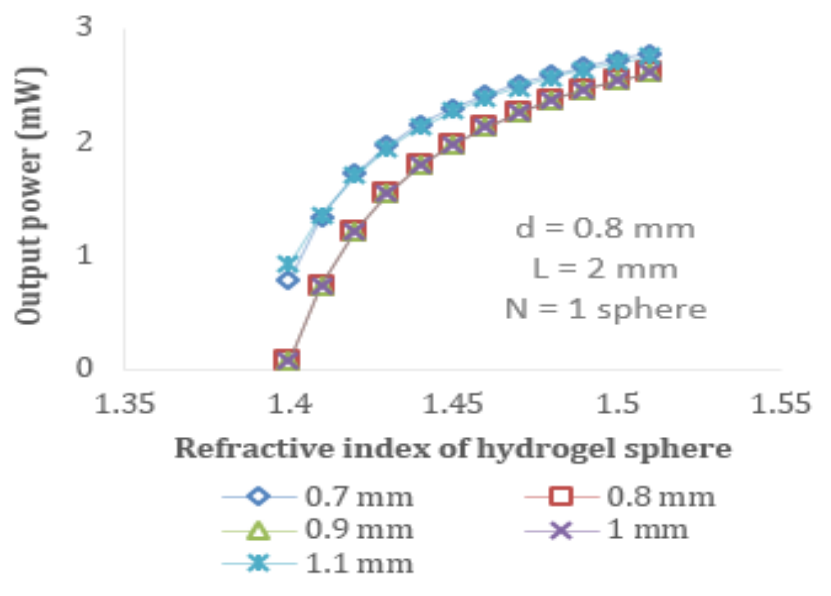

C

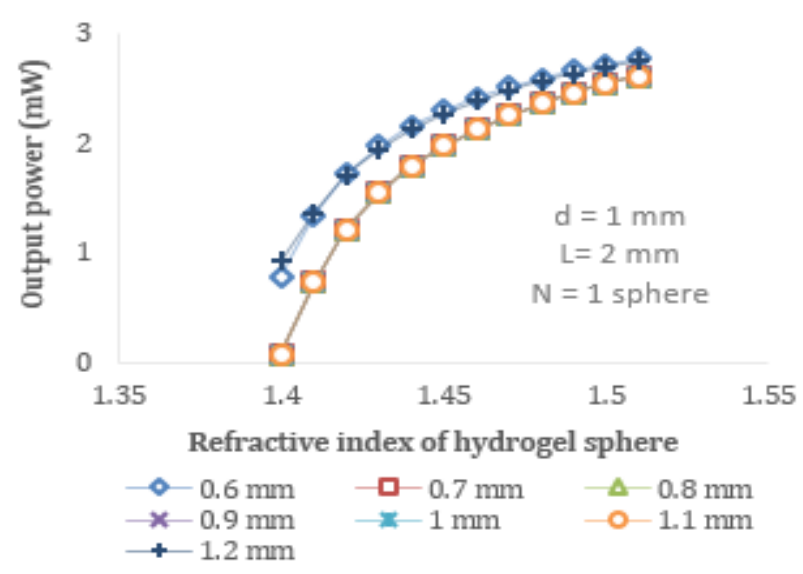

Figure 8: Sensor output power $(\mathrm{mW})$ vs. refractive index of hydrogel as a function of $a$ parameter for (A) $d=0.6 \mathrm{~mm},(B)$ $\mathrm{d}=0.8 \mathrm{~mm},(\mathrm{C}) \mathrm{d}=1 \mathrm{~mm}$.

The diagrams show that the sphere with diameter of 1 $\mathrm{mm}$ can be displaced to both sides around $a=0.9 \mathrm{~mm}$ with the step of $0.2 \mathrm{~mm}$, which resulting no alternations on the sensitivity. Thus, it can be said that by increasing the size of the sphere and enclosing a greater area of no-core, the amount of sensitivity variations resulting from displacement 
is reduced and the output is obtained with similar quantities. Therefore, the error of displacements arising from the experimental test will be decreased.

\subsection{The effect of the spheres number ( $N$ parameter)}

The last aspect that is studied in relation to spheres is their number corresponding to the applied lengths of the no-core fiber. For the purpose of understanding how it affects the sensitivity of the sensor, the desired changes in lengths of 2 , 4 and $7 \mathrm{~mm}$ of the no-core fiber are applied in accordance with the diagrams of Fig. 9. Therefore, the effect of the increasing number of the spheres with different lengths of the no-core fiber is observed. Simultaneously, besides the study of the number of that spheres, the influence of the separation distance between their centers ( $x$ parameter) has also been reviewed in the curves.

A

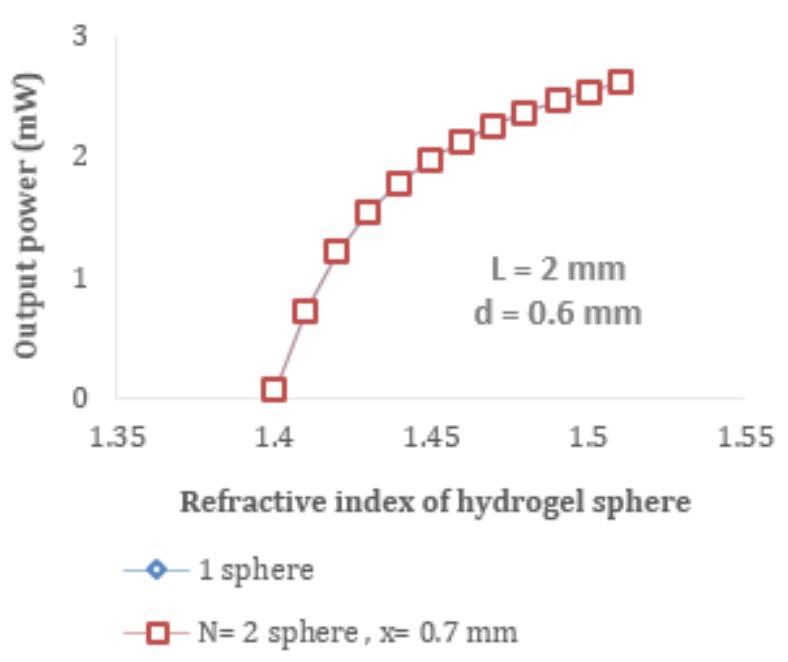

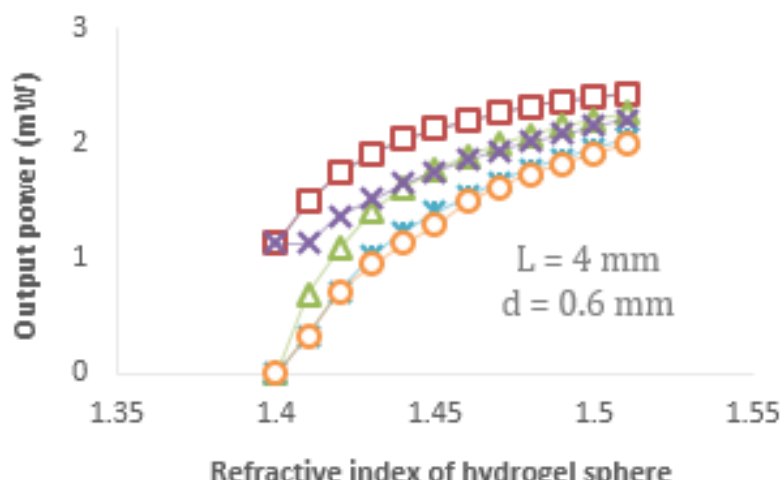

Refractive index of hydrogel sphere

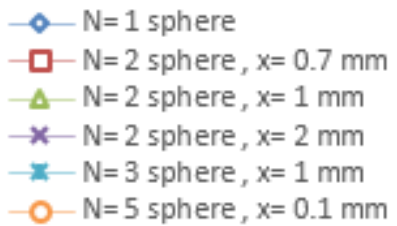

$\mathrm{C}$

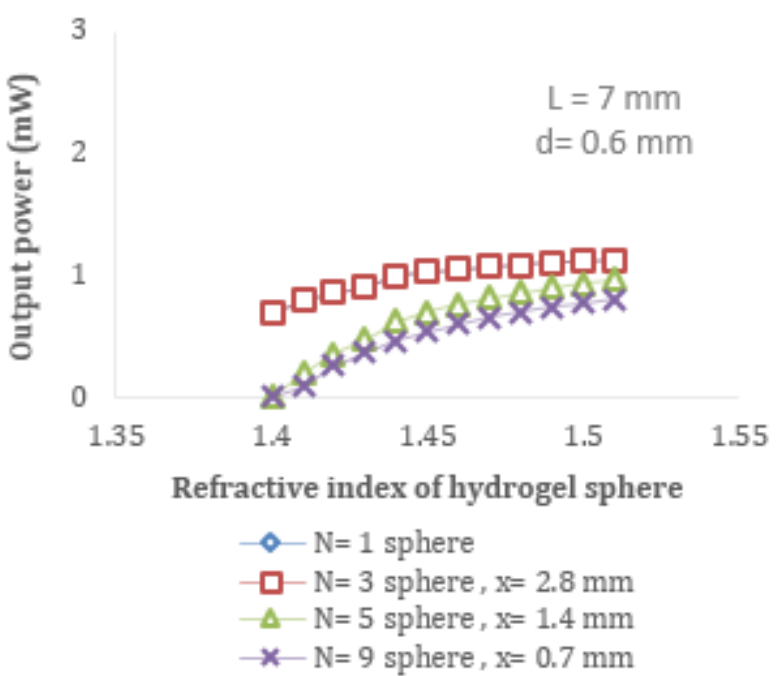

Figure 9: Sensor output power (mW) vs. refractive index of hydrogel as a function of $N$ parameter for (A) $L=2 \mathrm{~mm}$, (B) $L=4 \mathrm{~mm}$, (C) $L=7 \mathrm{~mm}$.

In Fig. 9, the charts related to the length of $4 \mathrm{~mm}$ show that by increasing the number of hydrogel spheres, the diagrams tend to be linearized and their sensitivity are improved, while in other lengths, no significant changes will be made. Hence, in Fig. 10, increasing the number of hydrogel spheres with diameter of $1 \mathrm{~mm}$ is also examined. Finally this experiment have been made with 3 hydrogel spheres, since there is no particular change in variations of output power when the number of spheres is increased from 3 to 5 . Among the three diagrams, the only chart related to the number of 3 spheres in the refractive index range of 1.42 to 1.51 , has been shown more linear and sensitive. 


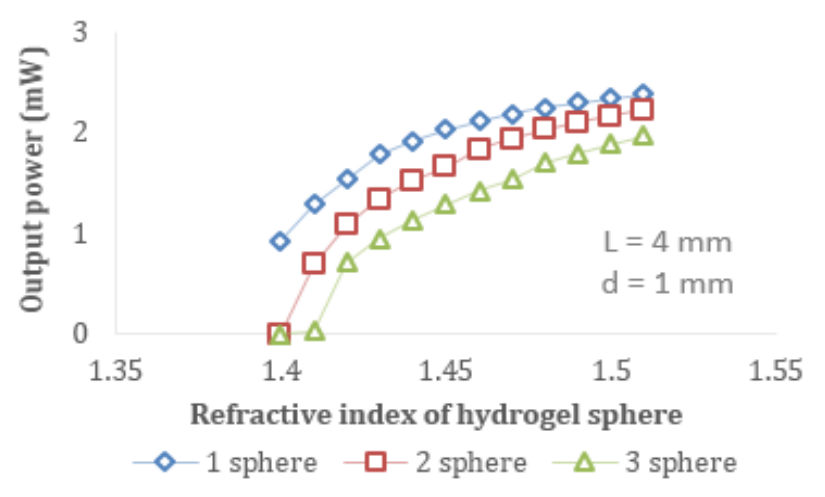

Figure 10: Sensor output power $(\mathrm{mW})$ vs. refractive index of hydrogel as a function of $N$ parameter.

\subsection{Ultimate structure from comparing the results}

After investigating all the above aspects and observing the obtained charts, the highest sensitivities result from the lengths of 2 and $4 \mathrm{~mm}$ which can even be improved by increasing the number of spheres over $4 \mathrm{~mm}$ no-core. Also, the slope goes from exponential to linear in their charts. In this section, all results of the simulations are compared and the only output which gives the highest sensitivity in a more linear manner with the maximum range of humidity is the structure of Fig. 11.

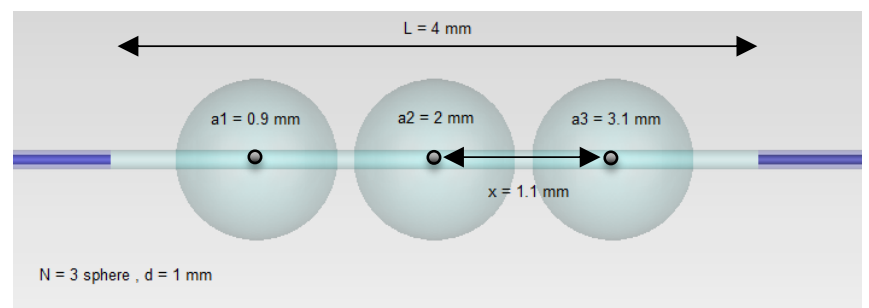

Figure 11: Ultimate structure of the sensor with the highest sensitivity.

According to Fig. 12, the approximation of the associated chart with the output of refractive indexes greater than 1.42 gives a line with a constant slope which is the average $\mathrm{RH}$ sensitivity of the sensor. The regression equation that can be calculated from this linear slope is:

$$
P(m W)=-13.643 R H+2.1914,
$$

Which includes the humidity range of $20-95 \% \mathrm{RH}$.

\section{Conclusions}

In this paper, it is tried to accurately and efficiently combine two previous methods into a new structure. Designed senor consists of a multi-mode fiber for transmitting light and a nocore fiber for increasing the leakage modes of light to the outside. The main advantage of this structure is due to the applied PEGDMA hydrogel spheres, instead of a thin coating to efficiently expose the light in interaction with humidity. Also, LED connection is used instead of laser as an input source and a detector with less resolution in output that all will reduce the cost of construction and makes it easier to fabricate. one can conclude from the simulations is about the most effective factors on the sensitivity of the sensor which includes the length of no-core fiber $(L)$, the number of hydrogel spheres $(N)$ and the location of the center of the first sphere $\left(a_{1}\right)$. In this way, reducing the length of the no-core fiber, increasing the number of the spheres which helps to linearize the curves and placing the first sphere on the part of the fiber with the highest light leakage $\left(a_{l}=0.9 \mathrm{~mm}\right)$ make the highest sensitivity of the sensor. By contrast, the factors that have the less effect on the sensitivity are the changes in the diameter of the spheres $(d)$ and the separation distance between each sphere $(x)$. Hence, it is possible to select a smaller size of sphere with a slight displacement, which reduces the gravity impact on the geometric deformation of hydrogel droplets to be elliptical and the displacement inducted error in the practical experiment. However, the simultaneous impact of several factors should not be ignored. For example, an increase in the number of the spheres over 2 $\mathrm{mm}$ no-core fiber does not have any influence on sensitivity, while its increase over $4 \mathrm{~mm}$ no-core fiber improves the sensitivity and its performance, and over the long lengths of the no-core fiber will have no effect.

Hence, in this structure the intended sensitivity for humidity in the range of 20 to $95 \% \mathrm{RH}$, is $0.13643 \mathrm{~mW} / \%$ $\mathrm{RH}$. In addition to its low cost, the sensor is capable of detecting a wide range of humidity, with small dimensions and length, despite the lack of enough improvement of sensitivity. Although it is tried to evaluate sensor function in order to ensure the accuracy and stability of the results of the simulations by considering the possible errors to eliminate them in practice, it is still necessary to examine the sensor experimentally. Also, sensor response time and performance can be improved by using more suitable hydrogels with better humidity sensitivity in the future.

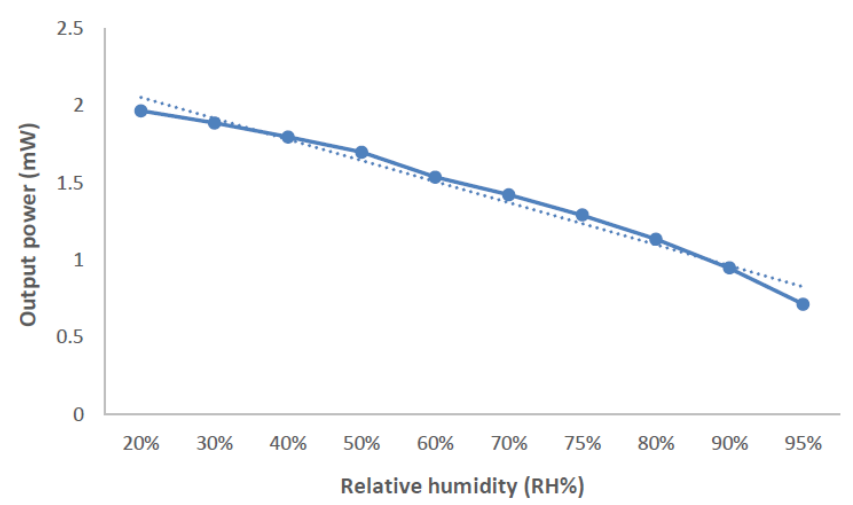

Figure 12: Designed sensor output power $(\mathrm{mW})$ Vs. relative humidity $(\% \mathrm{RH})$.

\section{References}

[1] L. Alwis, T. Sun, K.T.V. Grattan, Optical fiber-based sensor technology for humidity and moisture measurement: Review of recent progress. Measurement 46(10): 4052-4074, 2013. 
[2] Stanislav A.K., Neil T.G., Chengbo M., Kaiming Z., Toward a new generation of photonic humidity sensors. Sensors 14: 3986-4013, 2014.

[3] Saeed Azad, Ebrahim Sadeghi, Roghaieh Parvizi, Azardokht Mazaheri, M. Yousefi, Sensitivity optimization of $\mathrm{ZnO}$ clad-modified optical fiber humidity sensor by means of tuning the optical fiber waist diameter. Optics \& Laser Technology. 90: 96-101, 2017.

[4] Guofeng Yan, Yanhong Liang, El-Hang Lee, and Sailing He, Novel Knob-integrated fiber Bragg grating sensor with polyvinyl alcohol coating for simultaneous relative humidity and temperature measurement. Opt. Express 23, 15624-15634, 2015.

[5] A. Lokman, H. Arof, S.W. Harun, Tapered fiber coated with hydroxyethyl cellulose/polyvinylidene fluoride composite for relative humidity sensor. Sensors and Actuators A. 225: 128-132, 2015.

[6] Y. Luo, C. Chen, Kia Xia, S. Peng, H. Guan, J. Tang,... $\&$ Z. Chen, Tungsten disulfide (WS 2) based all-fiberoptic humidity sensor. Optics express 24(8): 8956-8966, 2016.

[7] Y. Xiao, J. Zhang, X. Cai, S. Tan, J. Yu, H. Lu,... \& Z. Chen, Reduced graphene oxide for fiber-optic humidity sensing. Optics express 22(25): 31555-31567, 2014.

[8] Yangzi Zheng, Xinyong Dong, Chunliu Zhao, Yi Li, Liyang Shao, Shangzhong Jin, Relative humidity sensor based on microfiber loop resonator. Advances in Materials Science and Engineering, 2013.

[9] H. Guan, K. Xia, C. Chen, Y. Luo, J. Tang, H. Lu,... \& Z. Chen, Tungsten disulfide wrapped on micro fiber for enhanced humidity sensing. Optical Materials Express 7(5): 1686-1696, 2017.

[10] Getinet Woyessa, Kristian Nielsen, Alessio Stefani, Christos Markos, Ole Bang, Temperature insensitive hysteresis free highly sensitive polymer optical fiber Bragg grating humidity sensor. Opt. Express 24: 12061213, 2016.

[11] Gilad Masri, Shir Shahal, Avi Klein, Hamootal Duadi, Moti Fridman, Polarization dependence of asymmetric off-resonance long period fiber gratings, Opt. Express 24: 29843-29851, 2016.

[12] Tao Li, X. Dong, C.C. Chan, Kai Ni, S. Zhang, P.P. Shum, Humidity sensor with coated photonic crystal fiber interferometer, IEEE Sens. J. 13(6): 2214-2216, 2013.

[13]Ran Gao. Yi Jiang, Wenhui Ding, Agarose gel filled temperature-insensitive photonic crystal fibers humidity sensor based on the tunable coupling ratio. Sens. Actuators B Chem. 195: 313-319, 2014.

[14] F.J. Arregui, Z. Ciaurriz, M. Oneca, I.R. Matıá s, An experimental study about hydrogels for the fabrication of optical fiber humidity sensors. Sens. Actuators $B$ Chem. 96: 165-172, 2003.

[15] M. Batumalay, A. Lokman, F. Ahmad, H. Arof, H. Ahmad, S.W. Harun, Tapered plastic optical fiber coated with HEC/PVDF for measurement of relative humidity. IEEE Sens. J 13: 4702-4705, 2013.

[16] S. Akita, H. Sasaki, K. Watanabe, A. Seki, A humidity sensor based on a hetero-core optical fiber. Sens. Actuators B Chem. 147: 385-391, 2010.

[17] Nianbing Zhong, Mingfu Zhao, and Yishan Li, Ushaped, double-tapered, fiber-optic sensor for effective biofilm growth monitoring, Biomed. Opt. Express 7: 335-351, 2016.

[18] B.Y. Yang, Y.X. Niu, B.W. Yang, Y.H. Hu, L.L. Dai, Y.H. Yin, M. Ding. High Sensitivity Curvature Sensor with Intensity Demodulation Based on Single-ModeTapered Multimode-Single-Mode Fiber. IEEE Sens. J. 18: 1094-1099, 2018.

[19] Zhi Feng Zhang, Yilei Zhang, Humidity sensor based on optical fiber attached with hydrogel spheres. Opt Laser Technol. 74: 16-19, 2015.

[20] C. Lv, H. Xia, Q. Shi, G. Wang, Y.-S. Wang, Q.-D. Chen, H.-B. Sun, Sensitively Humidity-Driven Actuator Based on Photopolymerizable PEG-DA Films. Adv. Mater. Interfaces 4: 1601002, 2017.

[21] C. Lv, X. C. Sun, H. Xia, Y. H. Yu, G. Wang, X. W. Cao, S. X. Li, Y. S. Wang, Q. D. Chen, Y. D. Yu, H. B. Sun, Humidity-responsive actuation of programmable hydrogel microstructures based on 3D printing. Sensors and Actuators B. 259: 736-744, 2018.

[22] L. Xia, L. Li, W. Li, T. Kou, D. Liu, Novel optical fiber humidity sensor based on a no-core fiber structure. Sens. Actuators A: Phys. 190: 1-5, 2013. 